\title{
On the Scholarship of Generalisation: Research at a Higher Level
}

\author{
Thomas Adams \\ Professor, Department of Mechanical Engineering \\ Rose-Hulman Institute of Technology \\ Terre Haute, IN, USA \\ adams1@rose-hulman.edu
}

In recent decades the advent of advanced software and continually increasing computational power has led to the highest degree of detail and accuracy in the modelling of physical systems and processes that has ever been seen. When the default strategy is to use such techniques, however, it can become difficult to discern physical trends, and important relationships between variables (or lack thereof) may be overlooked altogether. When engaged in the "scholarship of generalisation," as opposed to specialisation, the researcher purposely analyses processes at hierarchically higher conceptual levels in search of these connections. What may be lost in terms of accuracy in the resulting predictive models is regularly made up for by the vastly increased physical insight that is gained. Oftentimes interdisciplinary in nature, the broad-based approach of the scholarship of generalisation well-complements the traditionally deep and narrow perspective of specialisation. 